\title{
IDENTIFICATION OF A NONLINEAR POLYNOMIAL COMPARTMENTAL SYSTEM OF $(\alpha+\beta)$ ORDER BY A LINEARIZATION METHOD
}

\author{
B. HEBRI ${ }^{1}$ and Y. CHERRUAULT ${ }^{2}$
}

${ }^{1}$ Faculty of mathematics U.S.T.H.B.- BP $n^{\circ} 32$ - EL ALIA

Alger 16111 - Algéria

${ }^{2}$ Pierre et Marie Curie University, MEDIMAT

Paris, France

E-mail: bhebri@yahoo.fr; cherruau@ccr.ccr.jussieu.fr

Received March 9, 2005; revised April 19, 2006; published online May 25, 2006

\begin{abstract}
A linearization method is used for identifying a nonlinear polynomial compartmental system of $(\alpha+\beta)$ order. We bring back the nonlinear model to a linear one for which a method, developed for Michaelis-Menten systems in a previous paper, can be used.

Key words: Problem inverse, linear compartimental system, nonlinear compartmental system, identification, ordinary differential equation
\end{abstract}

\section{Introduction}

In general the compartimental systems are used in fields very varied such as medicine, biology, the chemistry. or the dynamics of the populations. Recently, Gian Italo Bischi [1] gave an application to the economic systems. The nonlinear systems occur particularly in dynamics of the populations. These systems are governed by the following law: "the flow from compartment $i$ to compartment $j$ is proportional to the expression $x_{i}^{\alpha} x_{j}^{\beta}$ " $(\beta=0$ if $j$ designates the system's outside) (see $[2,3,4]$ ). The proportionality parameters $k_{i j}$ denote the exchange parameters, $\alpha$ and $\beta$ are positive constants characterizing the compartmental system, and $x_{i}(t)$ designates the quantity in compartment $i$ at time $t$. These $k_{i j}$ characterize the exchanges between compartments. This law is said of $(\alpha+\beta)$ order.

Our aim is to study an inverse problem consisting in identifying the exchange parameters $k_{i j}$. As for Michaelis-Menten systems (see [7]) a linearization method is used. The linear model obtained in the neighbourhood of the 
initial condition $(a, 0)$ gives a bad interpretation of the physical phenomenon. A " temporization" is necessary for obtaining an exact interpretation of the phenomenon. Furthermore the nonhomogeneity problem due to the initial condition encountered in Michaelis-Menten systems implies that the deduced linear system is not always real. The measures given by the practitioners will be used in association with a temporization technique allowing to adapt the results obtained for identification in linear compartmental systems.

\section{Definitions and Notations}

We consider the nonlinear bicompartmental system of polynomial type, namely $\left(S_{N L}\right)$, shown in Figure 1.

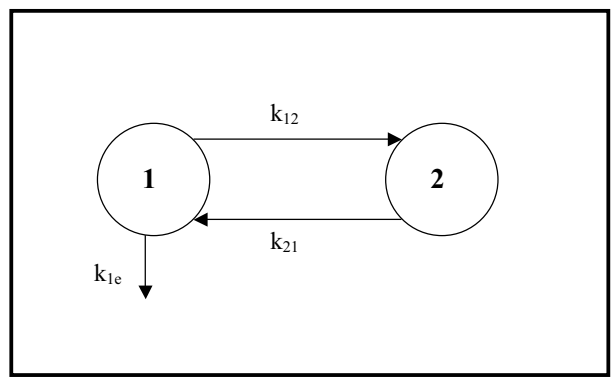

Figure 1. $\left(S_{N L}\right)$ : Nonlinear bicompartmental system.

The mass balance principle in each compartment leads to a nonlinear differential equations (see [2]). The identification is done by exciting the system with an instantaneous injection of substance quantity $a$ in the compartment 1 . Thus we can say that the compartmental system is governed by the following differential system with initial conditions :

$$
\left\{\begin{array}{l}
x_{1}^{\prime}(t)=k_{21} x_{2}^{\alpha}(t) x_{1}^{\beta}(t)-k_{12} x_{1}^{\alpha}(t) x_{2}^{\beta}(t)-k_{1 e} x_{1}^{\alpha}(t), \\
x_{2}^{\prime}(t)=k_{12} x_{1}^{\alpha}(t) x_{2}^{\beta}(t)-k_{21} x_{2}^{\alpha}(t) x_{1}^{\beta}(t), \\
x_{1}(0)=a, \quad x_{2}(0)=0 .
\end{array}\right.
$$

Let us set:

$$
\begin{aligned}
& X:\left[0,+\infty\left[\longrightarrow \mathbb{R}^{2},\right.\right. \\
& t \longrightarrow X^{T}(t)=\left(x_{1}(t), x_{2}(t)\right)
\end{aligned}
$$

the state function associated to compartmental system $\left(S_{N L}\right)$, and

$$
\begin{aligned}
& F: \mathbb{R}^{2} \longrightarrow \mathbb{R}^{2}, \\
& \left(x_{1}, x_{2}\right) \longrightarrow F\left(x_{1}, x_{2}\right)=\left(f_{1}\left(x_{1}, x_{2}\right), f_{2}\left(x_{1}, x_{2}\right)\right)
\end{aligned}
$$


the vectorial function defined by :

$$
\left\{\begin{array}{l}
f_{1}\left(x_{1}, x_{2}\right)=k_{21} x_{2}^{\alpha} x_{1}^{\beta}-k_{12} x_{1}^{\alpha} x_{2}^{\beta}-k_{1 e} x_{1}^{\alpha}, \\
f_{2}\left(x_{1}, x_{2}\right)=k_{12} x_{1}^{\alpha} x_{2}^{\beta}-k_{21} x_{2}^{\alpha} x_{1}^{\beta} .
\end{array}\right.
$$

With these notations we can write the differential system (2.1) under the vectorial form :

$$
\left\{\begin{array}{l}
X^{\prime}(t)=F(X(t)), \\
X(0)=\left(\begin{array}{l}
a \\
0
\end{array}\right) .
\end{array}\right.
$$

\section{Linearization of the Differential System}

The partial derivatives of the function $F$ are defined as follows:

$$
\begin{aligned}
& \frac{\partial f_{1}}{\partial x_{1}}\left(x_{1}, x_{2}\right)=\beta k_{21} x_{2}^{\alpha} x_{1}^{\beta-1}-\alpha k_{12} x_{1}^{\alpha-1} x_{2}^{\beta}-\alpha k_{1 e} x_{1}^{\alpha-1} k_{1 e} x_{1}^{\alpha}, \\
& \frac{\partial f_{1}}{\partial x_{2}}\left(x_{1}, x_{2}\right)=\alpha k_{21} x_{2}^{\alpha-1} x_{1}^{\beta}-\beta k_{12} x_{1}^{\alpha} x_{2}^{\beta-1}, \\
& \frac{\partial f_{2}}{\partial x_{1}}\left(x_{1}, x_{2}\right)=\alpha k_{12} x_{1}^{\alpha-1} x_{2}^{\beta}-\beta k_{21} x_{2}^{\alpha} x_{1}^{\beta-1}, \\
& \frac{\partial f_{2}}{\partial x_{2}}\left(x_{1}, x_{2}\right)=\beta k_{12} x_{1}^{\alpha} x_{2}^{\beta-1}-\alpha k_{21} x_{2}^{\alpha-1} x_{1}^{\beta} .
\end{aligned}
$$

The function $F$ is differentiable in all point $\left(x_{1}, x_{2}\right)$ such that $x_{1} \neq 0$ and $x_{2} \neq 0$ for all $\alpha>0$ and all beta $>0$, and the Jacobian matrix is given by:

$$
(D F)_{\left(x_{1}, x_{2}\right)}=\left(\begin{array}{ll}
\frac{\partial f_{1}}{\partial x_{1}} & \frac{\partial f_{1}}{\partial x_{2}} \\
\frac{\partial f_{2}}{\partial x_{1}} & \frac{\partial f_{2}}{\partial x_{2}}
\end{array}\right) .
$$

For linearizing the system (2.2) we apply the Taylor formula in the neighbourhood of the initial condition $(a, 0)$. Furthermore:

i) $F$ is not differentiable in $(a, 0)$ if $\alpha<1$ or $\beta<1$.

ii) If $\alpha \geq 1$ and $\beta \geq 1 F$ is differentiable in $(a, 0)$. The Taylor formula applied in neighbourhood of $(a, 0)$ leads to:

$$
\begin{aligned}
X^{\prime}(t)= & F^{T}(a, 0)+(D F)_{(a, 0)}\left(x_{1}-a, 0\right)^{T} \\
& +\frac{1}{2}\left(D^{2} F\right)\left(a+\theta\left(x_{1}-a\right), \theta x_{2}\right)\left(\left(x_{1}-a, 0\right)^{T}\right)^{2}, \quad 0<\theta<1 .
\end{aligned}
$$


The linear bicompartmental system approximating the nonlinear system $\left(S_{N L}\right)$ is given as follows:

$$
X^{\prime}(t)=F^{T}(a, 0)+(D F)_{(a, 0)}\left(x_{1}-a, 0\right)^{T} .
$$

Then the explicit formulation is

$$
\left\{\begin{array}{l}
x_{1}^{\prime}(t)=p_{21} x_{2}(t)-p_{12} x_{1}(t)-p_{1 e} x_{1}(t), \\
x_{2}^{\prime}(t)=p_{12} x_{1}(t)-p_{21} x_{2}(t) \\
x_{1}(0)=a, \quad x_{2}(0)=0 .
\end{array}\right.
$$

This means that this linear bicompartmental system has the following figure:



Figure 2. $\left(S_{L}\right)$ : A linear bicompartmental system.

with

$$
\left\{\begin{aligned}
p_{12} & =\frac{\partial f_{2}}{\partial x_{1}}(a, 0)=0, \\
p_{21} & =\frac{\partial f_{1}}{\partial x_{2}}(a, 0)=\left\{\begin{aligned}
0, & \text { if } \alpha \geq 1 \text { and } \beta>1, \\
-a^{\alpha} k_{12}, & \text { at } \beta=1, \\
a^{\beta} k_{21}, & \text { if } \alpha=1 \text { and } \beta>1
\end{aligned}\right.
\end{aligned}\right.
$$

$p_{12}=0$ involves that there is no exchange between compartment 1 and 2 . Moreover if $\alpha=1$ and $\beta>1$, then the proposed model is not real because $p_{21}<0$. So the initial condition $x_{2}(0)=0$ is not well adapted for applying the method of temporization.

We suggest to introduce a "temporization". It means that we "wait a moment $t^{*}$ " after injecting the quantity $a$ for permitting exchange in the system $\left(S_{N L}\right)$, and we measure the compartment 1 at this time $t^{*}$. Then for $t>t^{*}$ the system $\left(S_{N L}\right)$ is governed by the following Cauchy problem:

$$
\left\{\begin{array}{l}
x_{1}^{\prime}(t)=k_{21} x_{2}^{\alpha}(t) x_{1}^{\beta}(t)-k_{12} x_{1}^{\alpha}(t) x_{2}^{\beta}(t)-k_{1 e} x_{1}^{\alpha}(t), t>t^{*}, \\
x_{2}^{\prime}(t)=k_{12} x_{1}^{\alpha}(t) x_{2}^{\beta}(t)-k_{21} x_{2}^{\alpha}(t) x_{1}^{\beta}(t) \\
x_{1}\left(t^{*}\right)=a^{*}, \quad x_{2}\left(t^{*}\right)=b .
\end{array}\right.
$$


Generally, compartment 2 is not accessible to the measurement and thus $b$ is unknown. This differential system can be written under the vectorial form:

$$
\left\{\begin{array}{l}
X^{\prime}(t)=F^{T}\left(X^{T}(t)\right) \\
X^{T}\left(t^{*}\right)=\left(a^{*}, b\right)
\end{array}\right.
$$

$F$ being a regular function, we can apply the Taylor formula to $F$ in the neighbourhood of $\left(a^{*}, b\right)$. There exists a time $t_{0}$ sufficiently small such that the system (3.1) can be approached by the linear differential system with initial condition on the interval $\left[t^{*}, t_{0}\right]$ :

$$
\left\{\begin{array}{l}
X^{\prime}(t)=F^{T}\left(a^{*}, b\right)+(D F)_{\left(a_{*}, b\right)}\left(x_{1}-a_{*},\left(x_{2}-b\right)\right)^{T}, t>t^{*} \\
X^{T}\left(t^{*}\right)=\left(a^{*}, b\right)
\end{array}\right.
$$

where :

$$
F^{T}\left(a_{*}, b\right)=\left(k_{21} b^{\alpha} a_{*}^{\beta}-k_{12} a_{*}^{\alpha} b^{\beta}-k_{1 e} a_{*}^{\alpha},{ }_{12} a_{*}^{\alpha} b^{\beta}-k_{21} b^{\alpha} a_{*}^{\beta}\right) .
$$

The error due to the linearization will be studied in another paper.

\section{Reduction of the System (3.2) to the Canonical Form}

For applying results of $[5,6,7]$ relating to the linear systems, it is necessary to reduce the differential system (4.2) to the general form:

$$
Y^{\prime}(t)=A Y(t) .
$$

This form is said to be canonical form ( $A$ being a matrix of order 2$)$. We are going to operate in two steps:

First step:

Lemma 1. Suppose that the system $\left(S_{N L}\right)$ is open. If $t^{*}$ is chosen such that

$$
\alpha k_{21} b^{\alpha-1}-\beta k_{12} a_{*}^{\alpha} b^{\beta-1} \neq 0,
$$

then there exists a unique set $(\gamma, \delta)$ in $\mathbb{R}^{2}$ such that:

$$
(D F)_{\left(a_{*}, b\right)}\left(\begin{array}{l}
\gamma \\
\delta
\end{array}\right)=F\left(a_{*}, b\right)
$$

More precisely:

$$
\gamma=\frac{a_{*}}{\alpha}, \quad \delta=\frac{(\alpha-\beta) a_{*}^{\beta}}{\alpha} \frac{k_{21} b^{\alpha}}{\alpha k_{21} b^{\alpha-1} a_{*}^{\beta}-\beta k_{12} a_{*}^{\alpha} b^{\beta-1}} .
$$


Proof. Equation

$$
(D F)_{\left(a_{*}, b\right)}\left(\begin{array}{l}
\gamma \\
\delta
\end{array}\right)=F\left(a_{*}, b\right)
$$

is a linear algebraic system according to $(\gamma, \delta)$, whose determinant, namely $D_{1}$, is:

$$
D_{1}=\alpha k_{1 e} a_{*}^{\beta-1}\left(\alpha k_{21} b^{\alpha-1} a_{*}^{\beta}-\beta k_{12} a_{*}^{\alpha} b^{\beta-1}\right) .
$$

If the system is open $\left(k_{1 e} \neq 0\right)$, then $D_{1} \neq 0$ and consequently the previous algebraic system has a unique solution:

$$
\gamma=\frac{a_{*}}{\alpha}=\gamma^{*}
$$

We denote $\gamma=\gamma^{*}$ because it is calculable, and

$$
\delta=\frac{(\alpha-\beta) a_{*}^{\beta}}{\alpha} \frac{k_{21} b^{\alpha}}{\alpha k_{21} b^{\alpha-1} a_{*}^{\beta}-\beta k_{12} a_{*}^{\alpha} b^{\beta-1}} .
$$

\section{Second step:} form:

Previous Lemma permits to write the differential system (4.2) under the

$$
X^{\prime}(t)=(D F)_{\left(a^{*}, b\right)}\left(\begin{array}{c}
x_{1}(t)-a^{*}+\gamma \\
x_{2}(t)-b+\delta
\end{array}\right) .
$$

The change of the state variables

$$
Y(t)=\left(\begin{array}{l}
y_{1}(t) \\
y_{2}(t)
\end{array}\right)=\left(\begin{array}{c}
x_{1}(t)-a^{*}+\gamma_{*} \\
x_{2}(t)-b+\delta
\end{array}\right)
$$

reduces system (4.2) to its canonical form:

$$
\left\{\begin{array}{l}
Y^{\prime}(t)=(D F)_{\left(a^{*}, b\right)} Y(t), \\
Y^{T}\left(t^{*}\right)=\left(\gamma_{*}, \delta\right) .
\end{array}\right.
$$

Remark 1. The system $\left(S_{N L}\right)$ is approximated by the compartmental linear model, namely $\left(S_{C L}\right)$ shown in Figure 3, where

$$
p_{12}=\alpha k_{12} a_{*}^{\alpha-1} b^{\beta}-\beta k_{21} b^{\alpha} a_{*}^{\beta-1}, \quad p_{21}=\alpha k_{21} b^{\alpha-1} a_{*}^{\beta}-\beta k_{12} a_{*}^{\alpha} b^{\beta-1} .
$$

\section{Choice of the Initial Condition and Induced Problem}

The parameters $k_{12}, k_{21}$ and the constants $\alpha, \beta$ characterize the system. But the initial condition $a^{*}$ and $b$ depend on the choice of the time $t^{*}$. This involves that the signs of $p_{12}$ and $p_{21}$ are related to $t^{*}$ and are not known. To be sure 


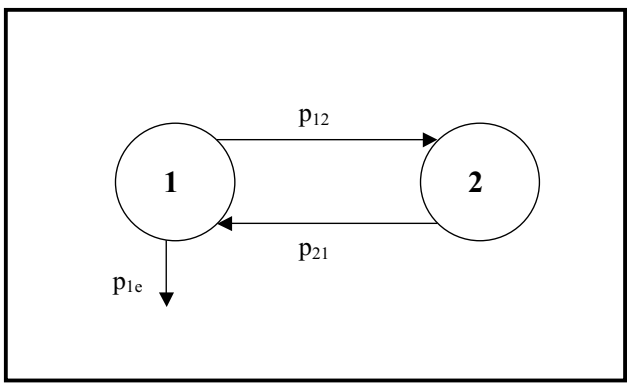

Figure 3. $\left(S_{C L}\right)$ : Approximation linear model.

that the linear model $\left(S_{C L}\right)$ corresponds to a measurable physical reality, $p_{12}$ and $p_{21}$ must be positive :

$$
\left\{\begin{array} { l } 
{ p _ { 1 2 } > 0 } \\
{ p _ { 2 1 } > 0 }
\end{array} \Leftrightarrow \left\{\begin{array}{l}
\alpha k_{12} a_{*}^{\alpha}-\beta k_{21} b^{\alpha-\beta} a_{*}^{\beta}>0 \\
\alpha k_{21} b^{\alpha-\beta} a_{*}^{\beta}-\beta k_{12} a_{*}^{\alpha}>0 .
\end{array}\right.\right.
$$

Two questions arise:

i) Do there exist values of $a_{*}$ and $b$ such that $\left\{\begin{array}{c}p_{12}>0, \\ p_{21}>0\end{array}\right.$ ?

ii) Moreover, $a_{*}$ and $b$ being tied, does it exist a couple $\left(a^{*}, b\right)$ satisfying the condition, or in other words, does it exist a value $t^{*}$ verifying this condition?

A first answer is given by the following

Proposition 1. For all value of $a_{*}$ fixed, $0<a_{*}<a$, there exists $b>0$ such that:

$$
p_{12}>0, p_{21}>0 \text { if and only if } \alpha>\beta \text {. }
$$

Proof. Set $x=k_{12} a_{*}^{\alpha}$ and $y=k_{21} b^{\alpha-\beta} a_{*}^{\beta} \quad(x>0$ and $y>0)$

$$
\left\{\begin{array} { l } 
{ p _ { 1 2 } > 0 } \\
{ p _ { 2 1 } > 0 }
\end{array} \Leftrightarrow \left\{\begin{array}{l}
\alpha x-\beta y>0 \\
\alpha y-\beta x>0
\end{array}\right.\right.
$$

If $\alpha<\beta$ the solutions set $\left\{\begin{array}{l}\alpha x-\beta y>0 \\ \alpha y-\beta x>0\end{array}\right.$ is empty.

If $\alpha>\beta$ the solutions set $\left\{\begin{array}{l}\alpha x-\beta y>0 \\ \alpha y-\beta x>0\end{array}\right.$ is not empty. 
Next we should check if the values found above are suitable. We are going to show that the choice of $t^{*}$ is compatible with one real system as soon as the eigenvalues $\lambda_{1}^{*}$ and $\lambda_{2}^{*}$ obtained by the minimization of an error functional (defined below) are negative.

The compartment 1 of the system $\left(S_{N L}\right)$ is measured at instants $t_{j}, 1 \leqslant$ $j \leqslant m$. Let us consider the functional:

$$
J_{\left(i_{1}, i_{p}\right)}\left(\beta_{1}^{1}, \beta_{2}^{1}, \lambda_{1}, \lambda_{2}\right)=\sum_{j=i_{1}}^{i_{p}}\left(x_{1}\left(t_{j}\right)-\left(\beta_{1}^{1} e^{\lambda_{1} t_{j}}+\beta_{2}^{1} e^{\lambda_{2} t_{j}}\right)\right)^{2},
$$

where $1 \leqslant i_{1} \leqslant m-1$ and $2 \leqslant i_{p} \leqslant m$. Parameters $i_{1}$ and $i_{p}$ are chosen such that:

$$
\min J_{\left(i_{1}, i_{p}\right)}\left(\beta_{1}^{1}, \beta_{2}^{1}, \lambda_{1}, \lambda_{2}\right)
$$

is realized for $\lambda_{1}<0$ and $\lambda_{2}<0$. As matter of fact let us prove the following proposition:

Proposition 2. Let $\lambda_{1}^{*}, \lambda_{2}^{*}, \beta_{1}^{1 *}$, and $\beta_{2}^{1 *}$ be the values such that:

$$
\min J_{\left(i_{1}, i_{p}\right)}\left(\beta_{1}^{1}, \beta_{2}^{1}, \lambda_{1}, \lambda_{2}\right)=J_{\left(i_{1}, i_{p}\right)}\left(\beta_{1}^{1 *}, \beta_{2}^{1 *}, \lambda_{1}^{*}, \lambda_{2}^{*}\right) .
$$

If $\lambda_{1}^{*}<0, \lambda_{2}^{*}<0, \beta_{1}^{1 *} \neq 0$ and $\beta_{2}^{1 *} \neq 0$, then $p_{12}>0, p_{21}>0$.

Proof. Note the compartmental matrix of the linear model $\left(S_{C L}\right)$

$$
A=\left(\begin{array}{cc}
-p_{1 e}-p_{12} & p_{21} \\
p_{12} & -p_{21}
\end{array}\right) \Rightarrow \operatorname{det} A=\lambda_{1}^{*} \lambda_{2}^{*}=p_{1 e} p_{21} .
$$

But $p_{1 e}=\alpha k_{1 e}$, thus $p_{1 e}>0$ and consequently $p_{21}>0$.

It is proved in [3] that

$$
p_{12}=-\frac{\left(\lambda_{1}^{*}+p_{1 e}\right)\left(\lambda_{2}^{*}+p_{1 e}\right)}{p_{1 e}}
$$

then, supposing that $\lambda_{2}^{*}<\lambda_{1}^{*}$, we get

$$
p_{12}>0 \Leftrightarrow\left(-\lambda_{1}^{*}\right)<p_{1 e}<\left(-\lambda_{2}^{*}\right) .
$$

According to [3] we have:

$$
\left(\beta_{1}^{1 *} \neq 0, \text { and } \beta_{2}^{1 *} \neq 0\right) \Rightarrow\left(-\lambda_{1}^{*}\right)<p_{1 e}<\left(-\lambda_{2}^{*}\right) .
$$

This proves the result.

Corollary 1. We can set $t^{*}=t_{i 1}$. 


\section{Identification of the Systems $\left(S_{C L}\right)$ and $\left(S_{N L}\right)$}

\subsection{Identification of the system $\left(S_{C L}\right)$}

The following hypothesis for identification of the linear nonhomogeneous compartmental systems shown in Figure 4 are satisfied (see [7]): the system is

- linear, open,

- nonhomogeneous,

- undeterminated,

- satisfying initial conditions

$$
\left\{\begin{array}{c}
y_{1}(0)=\frac{a_{*}}{\alpha}, \\
y_{2}(0)=\delta
\end{array}\right.
$$

We use a new variable $s=t-t^{*}$ and $\delta$ is unknown.

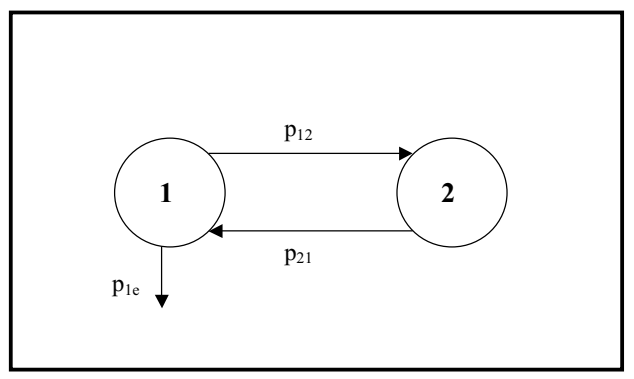

Figure 4. Linear nonhomogeneous bicompartmental system.

The excretion coefficient $p_{1 e}$ is identified (see [3]) by

$$
p_{1 e}=k_{1 e}=\frac{-\left(\lambda_{1}^{*}+\lambda_{2}^{*}\right)-\sqrt{\left(\lambda_{1}^{*}+\lambda_{2}^{*}\right)^{2}-8 \lambda_{1}^{*} \lambda_{2}^{*}}}{2},
$$

then the matrix of partial measures is completed as follows:

$$
\left\{\begin{array}{l}
\beta_{1}^{2}=-\left(1+\frac{p_{1 e}}{\lambda_{1}^{*}}\right) \beta_{1}^{1 *}, \\
\beta_{2}^{2}=-\left(1+\frac{p_{1 e}}{\lambda_{2}^{*}}\right) \beta_{2}^{1 *}
\end{array}\right.
$$

and consequently the exchange parameters $p_{12}$ and $p_{21}$ are identified by solving a linear algebraic system giving identification (see Hebri, B. \& Cherruault, Y. 2002a [6, 7]). Let: 


$$
\left\{\begin{array}{l}
\nu_{2}^{*}=p_{12}=\alpha k_{12} a_{*}^{\alpha-1} b^{\beta}-\beta k_{21} b^{\alpha} a_{*}^{\beta-1}, \\
\nu_{3}^{*}=p_{21}=\alpha k_{21} b^{\alpha-1} a_{*}^{\beta}-\beta k_{12} a_{*}^{\alpha} b^{\beta-1}
\end{array}\right.
$$

be this solution, and set $\nu_{1}^{*}=p_{1 e}$. This notation will be used in the next section.

\subsection{Identification of the nonlinear system $\left(S_{N L}\right)$}

For obtaining an approximation of the non linear system $\left(S_{N L}\right)$, it suffices to determine $b$.

Proposition 3. Let

$$
B^{* 2}=\left(\begin{array}{cc}
\beta_{1}^{1 *} & \beta_{1}^{2 *} \\
\beta_{2}^{1 *} & \beta_{2}^{2 *}
\end{array}\right)
$$

be the completude of the partial measures matrix of the algebraic masses associated to the model $\left(S_{C L}\right)$. We suppose that this system is identified. Then the initial condition $b$ is obtained by the relationship:

$$
b=\frac{1}{\nu_{3}^{*}}\left[(\alpha+\beta)\left(-\lambda_{1}^{*} \beta_{1}^{2 *}-\lambda_{2}^{*} \beta_{2}^{2 *}\right)+a_{*} \nu_{2}^{*}\right] .
$$

Proof. $\quad\left(S_{C L}\right)$ being identified, the coefficients $k_{12}$ and $k_{21}$ verify

$$
\left\{\begin{array}{l}
\nu_{2}^{*}=p_{12}=\alpha k_{12} a_{*}^{\alpha-1} b^{\beta}-\beta k_{21} b^{\alpha} a_{*}^{\beta-1} \\
\nu_{3}^{*}=p_{21}=\alpha k_{21} b^{\alpha-1} a_{*}^{\beta}-\beta k_{12} a_{*}^{\alpha} b^{\beta-1}
\end{array}\right.
$$

We deduce that

$$
\begin{aligned}
b \nu_{3}^{*}-a_{*} \nu_{2}^{*} & =(\alpha+\beta)\left(k_{21} b^{\alpha} a_{*}^{\beta}-k_{12} a_{*}^{\alpha} b^{\beta}\right) \\
& =(\alpha+\beta) x_{2}^{\prime}(0) \\
& =(\alpha+\beta)\left(-\lambda_{1}^{*} \beta_{1}^{2 *}-\lambda_{2}^{*} \beta_{2}^{2 *}\right) .
\end{aligned}
$$

In conclusion, we have

$$
b=\frac{1}{\nu_{3}^{*}}\left[(\alpha+\beta)\left(-\lambda_{1}^{*} \beta_{1}^{2 *}-\lambda_{2}^{*} \beta_{2}^{2 *}\right)+a_{*} \nu_{2}^{*}\right] .
$$

Theorem 1. Let $\left(S_{N L}\right)$ be a nonlinear polynomial system, and $\left(S_{C L}\right)$ the linear associated model. If $\alpha>\beta$ an approximation of the parameters of $\left(S_{N L}\right)$ are given by

$$
\left\{\begin{array}{l}
k_{12}=\frac{\alpha \nu_{2}^{*} a_{*}+\beta \nu_{3}^{*} b}{\left(\alpha^{2}-\beta^{2}\right) a_{*}^{\beta} b^{\alpha}}, \\
k_{21}=\frac{\alpha \nu_{3}^{*} b+\beta \nu_{2}^{*} a_{*}}{\left(\alpha^{2}-\beta^{2}\right) a_{*}^{\beta} b^{\alpha}},
\end{array}\right.
$$

where $\nu_{2}^{*}$ and $\nu_{3}^{*}$ are the coefficients of $\left(S_{C L}\right)$. 
Proof. If $\alpha>\beta$ we can approach the nonlinear system $\left(S_{N L}\right)$ by the real linear model $\left(S_{C L}\right)$ shown in Figure 3 . This system is identified by

$$
\left\{\begin{array}{l}
p_{12}=\nu_{2}^{*}, \\
p_{21}=\nu_{3}^{*},
\end{array}\right.
$$

$b$ being determinated by the relationship (6.1). $S_{\text {alg }}$ is a linear algebraic system according to $\left(k_{12}, k_{21}\right)$ whose determinant namely $D_{S \text { alg }}$ is

$$
D_{S \text { alg }}=\left(\alpha^{2}-\beta^{2}\right)\left(a_{*} b\right)^{\alpha+\beta-1} \neq 0 .
$$

Then $S_{\text {alg }}$ admits a unique solution $\left(k_{12}, k_{21}\right)$ given by

$$
\left\{\begin{array}{l}
k_{12}=\frac{\alpha \nu_{2}^{*} a_{*}+\beta \nu_{3}^{*} b}{\left(\alpha^{2}-\beta^{2}\right) a_{*}^{\beta} b^{\alpha}}, \\
k_{21}=\frac{\alpha \nu_{3}^{*} b+\beta \nu_{2}^{*} a_{*}}{\left(\alpha^{2}-\beta^{2}\right) a_{*}^{\beta} b^{\alpha}} .
\end{array}\right.
$$

\section{Conclusion}

The linear model associated to the nonlinear polynomial compartmental system of $(\alpha+\beta)$ order involves three important difficulties :

i ) The initial condition at time $t=0$ does not permit to give a complete information about the model $\left(S_{N L}\right)$. A "temporization $t^{*}$ " is introduced to suppress this difficulty.

ii) If this temporization is not modulated, the linear model is not necessarily real. We have shown that the measures done on the compartment 1 permit to choose one measure at instant $t_{i_{1}}=t^{*}$ such that we can develop a linearization method as for the Michaelis-Menten system (see [3]).

iii) The nonhomogeneous condition $x_{2}\left(t^{*}\right)=b$ being unknown is identified from measures done on compartment 1 .

The error on the system's coefficients due to the linearization will be developed in another paper.

\section{References}

[1] G.I. Bischi. Compartmental analysis of economic systems with heterogeneous agents: an introduction. In: Beyond the Representative Agent. Elgar Pub. Co, 181-214, 1998.

[2] Y. Cherruault. Mathematical Modelling in Biomedicine. Optimal control of Biomedical Systems. Kluwer (Reidel), Dordrecht, 1986. 
[3] Y. Cherruault. Modèles et méthodes mathématiques pour les sciences du vivant. Presses Universitaires de France, (P.U.F) Paris, 1998.

[4] Y. Cherruault. Optimisation: méthodes locales et globales. Presses Universitaires de France, (P.U.F), Paris, 1999.

[5] B. Hebri and Y. Cherruault. Contribution to identification of linear compartmental systems. Kybernetes, 33(8), 1277-1291, 2004.

[6] B. Hebri and Y. Cherruault. Direct identification of general linear compartmental systems by means of $(n-2)$ compartments measures. Kybernetes, 34(7-8), 969982, 2005.

[7] B. Hebri and Y. Cherruault. New results about identifiability of linear open bicompartmental homogeneous system and identification of open MichaelisMenten system by a linear approach. Kybernetes, 34(7-8), 1159-1186, 2005. 